\title{
Sensitivity and specificity of chest computed tomography scan based on RT-PCR in COVID-19 diagnosis
}

\author{
Alireza Mirahmadizadeh ${ }^{1, A}$, Zahra Pourmontaseri, ${ }^{2, \mathrm{D}, \mathrm{E}}$, Sima Afrashteh ${ }^{3, \mathrm{E}}$, Mehrnaz Hosseinzadeh ${ }^{4, \mathrm{E}}$, \\ Jalal Karimi ${ }^{2, D}$, Mehdi Sharafi, ${ }^{3,5, B, C, D}$ \\ ${ }^{1}$ Noncommunicable Diseases Research Center, Department of Epidemiology, School of Health, Shiraz University of Medical Sciences, Shiraz, Iran \\ ${ }^{2}$ Department of Infectious Diseases, School of Medicine, Fasa University of Medical Science, Fasa, Iran \\ ${ }^{3}$ Student Research Committee, Shiraz University of Medical Sciences, Shiraz, Iran \\ ${ }^{4}$ Research Committee, Fasa University of Medical Sciences, Fasa, Iran \\ ${ }^{5}$ Non-Communicable Diseases Research Center, Fasa University of Medical Sciences, Fasa, Iran
}

\section{Abstract}

Purpose: COVID-19 is a novel, severely contagious and progressive infection occurring worldwide. The diagnosis of the disease is based on real-time polymerase chain reaction (RT-PCR) and computed tomography (CT) scan, even though they are still controversial methods.

Material and methods: We studied 54 patients with suspected COVID-19 and the two mentioned methods were compared with each other.

Results: Sensitivity and specificity of the abnormal chest CT scan, ground-glass opacity (GGO), consolidation opacity, and both of GGO and consolidation were also surveyed based on RT-PCR. The results showed that RT-PCR assay was negative in $23(42.6 \%)$ patients and positive in 31 (57.4\%) cases. Also, the patients with an abnormal chest CT scan comprised 37 (68.5\%). The sensitivity and specificity of abnormal CT scan were $78.6 \%$ and $42.3 \%$, respectively, based on the RT-PCR method.

Conclusions: Other techniques alongside CT scan and RT-PCR are advocated for accuracy of the COVID-19 diagnosis.

Key words: RT-PCR, sensitivity and specificity, COVID-19, chest CT scan.

\section{Introduction}

Seven years after the MERS epidemic in the world, a new disease has been identified in China that has been announced as an international emergency worldwide by the World Health Organization (WHO) [1]. Although China was originally the center of the disease, the virus quickly spread to many parts of the world, leading to tremendous mortality [2]. This novel virus belongs to the coronavirus family causing flu-like symptoms as well as SARS and MERS. The most important forms of transmission are droplets and physical contact [3]. On February 11, 2020, the WHO named the new virus coronavirus disease 2019 (COVID-19) [4]. The clinical manifestation of the disease include fever, cough and dyspnea representing pneumonia, acute respiratory syndrome and death in severe disease [5]. The risk factors of infection include diabetes mellitus, hypertension, and cardiovascular disease [6,7]. The number of COVID-19 patients is still rising, with 22,524,291 infected worldwide on April 22, 2020 [8].

The gold standard test for the disease is known as real-time polymerase chain reaction (RT-PCR) assay, but false-negative results may be reported due to the low viral load of samples and manner of sampling. On the other

Correspondence address:

Dr. Mehdi Sharafi, Research Committee, Shiraz University of Medical Sciences, Shiraz, Iran, e-mail:mehdisharafi_2002@yahoo.com

Authors' contribution:

A Study design · B Data collection · C Statistical analysis · D Data interpretation - E Manuscript preparation · F Literature search · G Funds collection 
hand, the chest computed tomography (CT) scan is employed as an important diagnostic supplement to verify the COVID-19 disease [9]. The advantages of this method are being more time-saving than RT-PCR [10], and high sensitivity (97\%) in early diagnosis [11-13]. Nevertheless, positive RT-PCR may occur in normal chest CT in COVID-19 patients [14]. Furthermore, the chest CT scans of COVID-19 patients have shown low positive predictive value [15].

According to the rapid spread of the disease and the importance of preventing and controlling COVID-19, the primary diagnosis is crucial. This study was conducted to compare between CT scan and RT-PCR test in patients suspected to have COVID-19 infection. Sensitivity and specificity of CT scan were also evaluated based on RT-PCR.

\section{Material and methods}

This study was a cross sectional design on patients with COVID-19 admitted to Vali-e-Asr Fasa Hospital. Sensitivity and specificity of the chest CT scan, and oropharyngeal and nasopharyngeal RT-PCR were considered in 54 suspected COVID-19 patients. Demographic information including sex and age was also collected. Chest CT scan results were categorized as ground-glass, consolidation and normal. Cohen's kappa coefficient was considered for RT-PCR and CT scan.

\section{Results}

Of 54 patients, 27 (50\%) were female and half were male, with the mean age range of $51.75 \pm 18.63$.

Table 1. Results of real-time polymerase chain reaction (RT-PCR), chest computed tomography (CT) scan and also sensitivity and specificity of chest CT scan based on RT-PCR test are presented for diagnosis of COVID-19

\begin{tabular}{|c|c|c|c|c|}
\hline \multirow[t]{2}{*}{ Variable } & \multicolumn{2}{|c|}{ RT-PCR results } & \multirow[t]{2}{*}{$\kappa$} & \multirow[t]{2}{*}{$p$-value } \\
\hline & Negative number (\%) & Positive number (\%) & & \\
\hline \multicolumn{5}{|c|}{ Ground glass opacity } \\
\hline No & $14(25.9)$ & $9(16.7)$ & \multirow[t]{2}{*}{0.218} & \multirow[t]{2}{*}{0.1} \\
\hline Yes & $12(22.2)$ & $19(35.2)$ & & \\
\hline Sensitivity & \multicolumn{4}{|c|}{$(67.9)$} \\
\hline Specificity & \multicolumn{4}{|c|}{$(53.8)$} \\
\hline False positive & \multicolumn{4}{|c|}{ (38.7) } \\
\hline False negative & \multicolumn{4}{|c|}{ (39.1) } \\
\hline \multicolumn{5}{|l|}{ Consolidation } \\
\hline No & $17(31.5)$ & $18(33.3)$ & \multirow[t]{2}{*}{0.011} & \multirow[t]{2}{*}{0.933} \\
\hline Yes & $9(16.7)$ & $19(35.2)$ & & \\
\hline Sensitivity & \multicolumn{4}{|c|}{$(35.7)$} \\
\hline Specificity & \multicolumn{4}{|c|}{$(65.4)$} \\
\hline False positive & \multicolumn{4}{|c|}{$(47.4)$} \\
\hline False negative & \multicolumn{4}{|c|}{$(51.4)$} \\
\hline \multicolumn{5}{|c|}{ Ground glass and consolidation } \\
\hline No & $11(36.7)$ & $6(20.0)$ & \multirow[t]{2}{*}{0.167} & \multirow[t]{2}{*}{0.09} \\
\hline Yes & $6(20.0)$ & $7(23.3)$ & & \\
\hline Sensitivity & \multicolumn{4}{|c|}{$(53.8)$} \\
\hline Specificity & \multicolumn{4}{|c|}{$(64.7)$} \\
\hline False positive & \multicolumn{4}{|c|}{$(35.3)$} \\
\hline False negative & \multicolumn{4}{|c|}{$(46.2)$} \\
\hline \multicolumn{5}{|c|}{ Abnormal CT accumulatively } \\
\hline No & $11(20.2)$ & $6(11.1)$ & \multirow[t]{2}{*}{0.211} & \multirow[t]{2}{*}{0.09} \\
\hline Yes & $15(27.8)$ & $22(40.7)$ & & \\
\hline Sensitivity & \multicolumn{4}{|c|}{$(78.6)$} \\
\hline Specificity & \multicolumn{4}{|c|}{$(42.3)$} \\
\hline False positive & \multicolumn{4}{|c|}{$(40.5)$} \\
\hline False negative & \multicolumn{4}{|c|}{$(35.3)$} \\
\hline
\end{tabular}


RT-PCR assay was carried out on the 54 suspected COVID-19 patients with the subsequent result of $23(42.6 \%)$ negative and 31 (57.4\%) positive cases.

The patients with an abnormal chest CT scan comprised 37 (68.5\%), while normal chest CT results were obtained in 17 (31.5\%) of the patients. 23/54 (42.6\%) patients showed ground-glass opacity (GGO), while $28 / 54$ (51.85\%) cases were with consolidation opacities. Patients demonstrating both GGO and consolidation opacities comprised 13/54 (24.1\%) in chest CT scan.

The sensitivity and specificity of chest CT scan based on the RT-PCR test (as a gold standard) for COVID-19 are presented in Table 1 . The sensitivity and specificity of abnormal CT scan were $78.6 \%$ and $42.3 \%$, respectively, based on the RT-PCR method. In the cases with GGO, the sensitivity and specificity of CT scan were calculated as $67.9 \%$ and $53.8 \%$ respectively, whilst CT scan findings of consolidated lungs showed $35.7 \%$ sensitivity and $65.4 \%$ specificity based on RT-PCR. These amounts were $53.8 \%$ sensitivity and $64.7 \%$ specificity for the simulations of GGO and consolidation opacities in suspected COVID-19 patients. CT scan images according to PCR findings are presented in Figure 1.
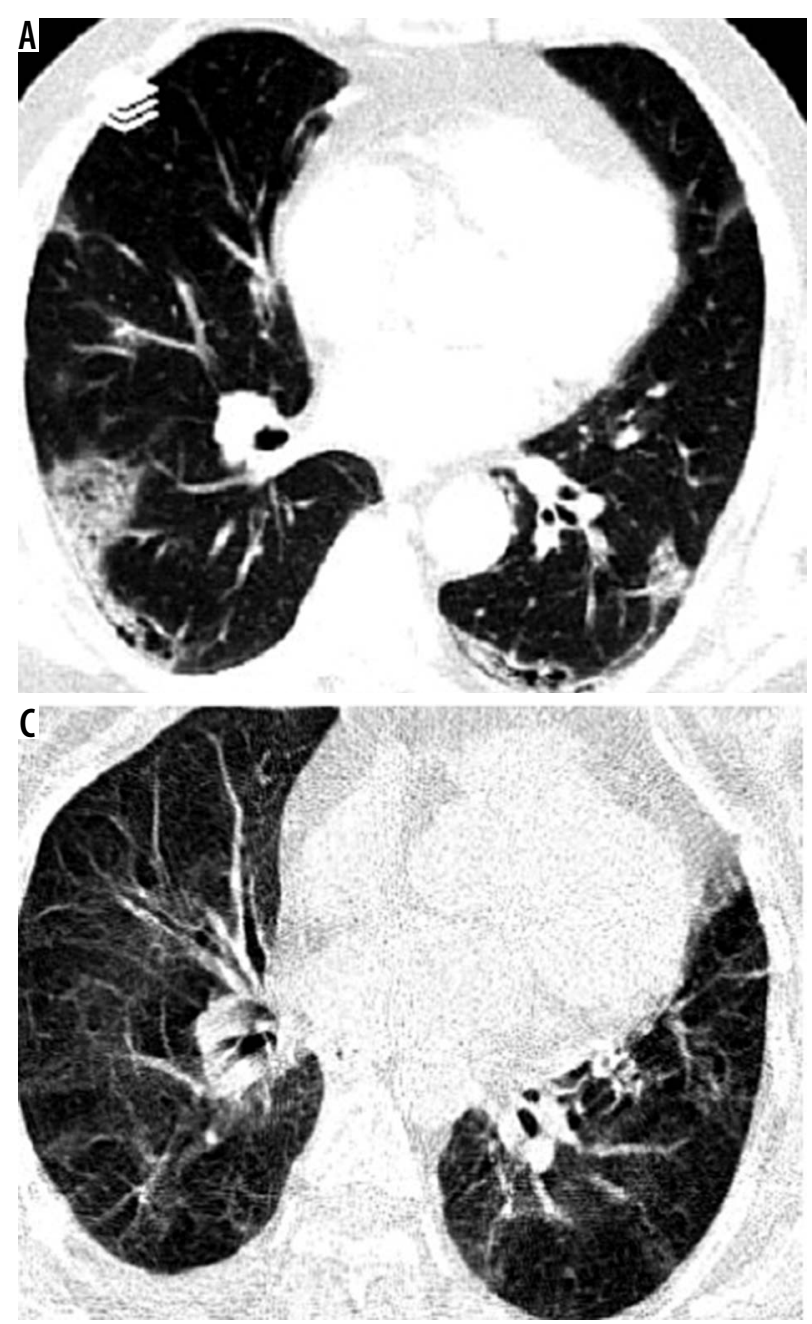

\section{Discussion}

Analyzed data of $54(f=m)$ suspected COVID-19 patients, $42.6 \%(n=23)$ showed GGO on chest CT; $57.4 \%$ $(n=31)$ had consolidation, $24.1 \%$ had both and only $16.6 \%$ had normal chest CT findings. $87.4 \%$ of the suspected cases tested positive in RT-PCR, reporting as the principal and accepted method in diagnosing COVID-19.

Our data on sensitivity and specificity of ground glass opacity showed $67.9 \%$ and $53.8 \%$ respectively ( $\kappa=0.218$ ). For chest CT with only consolidation, the sensitivity was $35.7 \%$ with the specificity of $65.4 \%(\kappa=0.11)$, and for the both GGO and consolidation the sensitivity and specificity were found to be $53.8 \%$ and $64.7 \%$ respectively in diagnosing COVID-19, based on RT-PCR.

Data on the correlation of chest CT and RT-PCR in COVID-19 diagnosis are located at the two extremes. Some studies, especially those from the radiology community, support a pivotal role for chest CT in diagnosing COVID-19. A report of 1014 patients from China proposed $97 \%$ sensitivity and specificity of $25 \%$ of CT scans based on RT-PCR results [11]. Another study from Wuhan, China, tried to determine the misdiagnosis rate of

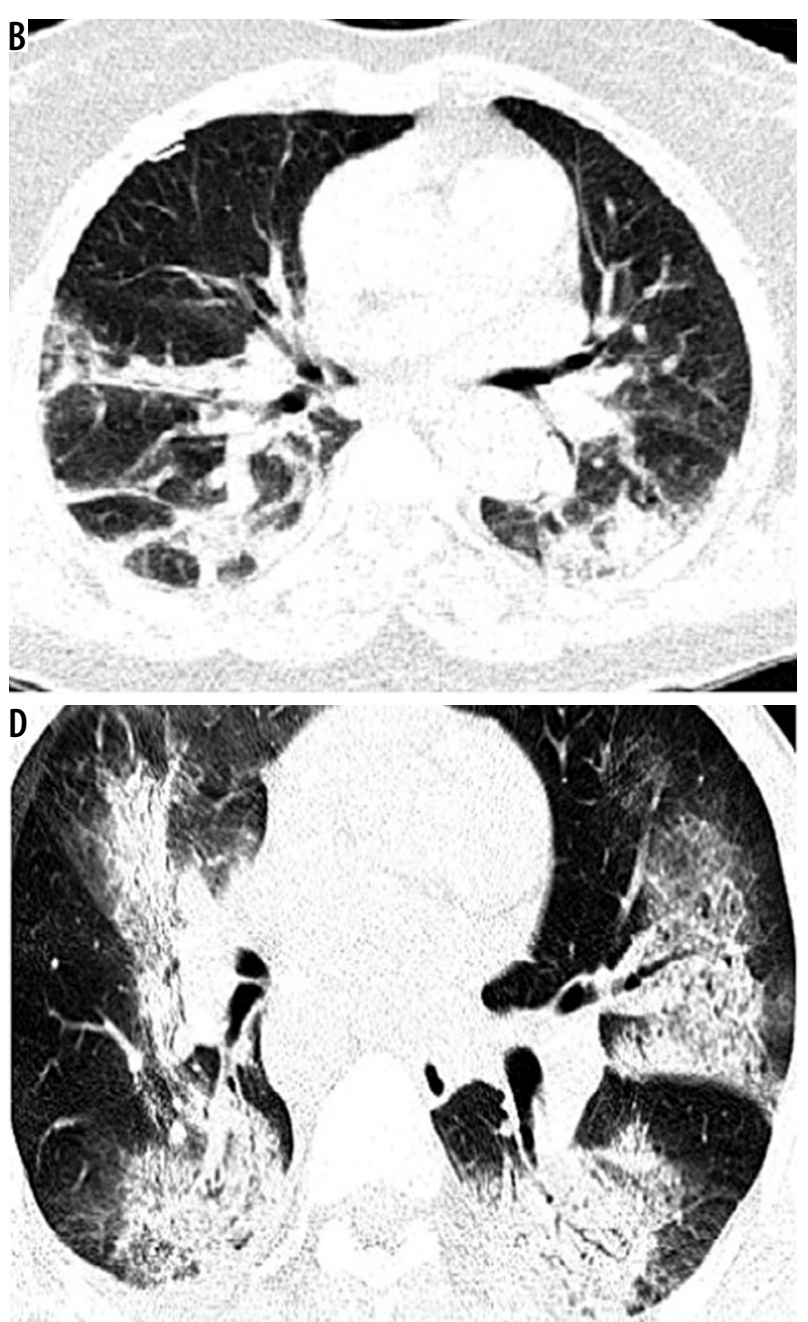

Figure 1. A) Ground-glass opacity (GG0) feature in computed tomography (CT) scan and polymerase chain reaction (PCR) positive case. B) GG0 consolidation in CT scan and PCR positive case. C) GG0 in CT scan and PCR negative case. D) GG0 consolidation and PCR negative case 
chest CT; among 51 confirmed cases of COVID-19, 3.9\% were found to be missed diagnoses. They suggested the use of chest CT as the standard method for screening and diagnosis of the disease [16]. Predominant findings in the CT scan of suspected patients were ground glass opacities and consolidation, local and bilateral patchy shadowing, and also crazy-paving patterns detected and associated with the disease [17-19].

In the extremely progressive outbreak of the COVID-19 pandemic, an accessible, accurate, sensitive, and specific diagnostic method is crucial. However, the RT-PCR kits, despite their high specificity and acceptable sensitivity, are not accessible worldwide. Chest CT scans, based on promising reports mostly from China, have been advocated, but are criticized. It is suggested that the CT findings of COVID-19 lack sufficient specificity to the disease, and they have been seen in a variety of infectious and noninfectious conditions. High specificity reports of CT scan in China may be due to the high incidence, while they are likely to be different in other regions with a lower incidence of the disease. Meanwhile, in seasonal flu (ground glass opacity and consolidation as chest patterns of diagnosis as well as H1N1 influenza, MERS, and SARS), the sensitivity of the chest CT scan would not be accurate enough. However, the criticizers also reported some scientific flaws in the previous discussed articles. The American College of Radiology and the Centers for Disease Control do not currently suggest chest CT for diagnosis of COVID-19 [20-22].

We suggest that a proper diagnosis of COVID-19 should be based on a mixture of clinical manifestation, nucleotide viral testing, history of contact and occupational and social exposure. Other techniques alongside CT scan and RT-PCR are also advocated for accuracy of the disease diagnosis. Moreover, considering the low diagnostic value of the chest CT scan may help the health care providers to determine the severity and course of the disease.

\section{Conflict of interest}

The authors report no conflict of interest.

\section{References}

1. Ye Z, Zhang Y, Wang Y, et al. Chest CT manifestations of new coronavirus disease 2019 (COVID-19): a pictorial review. Eur Radiol 2020; 30: 4381-4389.

2. Vashist SK. In vitro diagnostic assays for COVID-19: recent advances and emerging trends. Diagnostics (Basel) 2020; 10: 202.

3. Xiong Y, Sun D, Liu Y, et al. Clinical and high-resolution CT features of the COVID-19 infection: comparison of the initial and follow-up changes. Invest Radiol 2020; 55: 332-339.

4. Hassan S, Sheikh FN, Jamal S, et al. Coronavirus (COVID-19): a review of clinical features, diagnosis, and treatment. Cureus 2020; 12: e7355.

5. Talebpour M, Hadadi A, Oraii A, et al. Rationale and design of a registry in a Referral and Educational Medical Center in Tehran, Iran: Sina Hospital Covid-19 registry (SHCo-19R). Adv J Emerg Med 2020; 4: e53.

6. Huang X, Wei F, Hu L, et al. Epidemiology and clinical characteristics of COVID-19. Arch Iran Med 2020; 23: 268-271.

7. Verity R, Okell LC, Dorigatti I, et al. Estimates of the severity of coronavirus disease 2019: a model-based analysis. Lancet Infect Dis 2020; 20: 669-677.

8. Worldometer. COVID-19 coronavirus pandemic 2020. Available from: https://www.worldometers.info/coronavirus/.

9. Hani C, Trieu NH, Saab I, et al. COVID-19 pneumonia: a review of typical CT findings and differential diagnosis. Diagn Interv Imaging 2020; 101: 263-268.

10. Long C, Xu H, Shen Q, et al. Diagnosis of the coronavirus disease (COVID-19): rRT-PCR or CT? Eur J Radiol 2020; 126: 108961.

11. Ai T, Yang Z, Hou H, et al. Correlation of chest CT and RT-PCR testing in coronavirus disease 2019 (COVID-19) in China: a report of 1014 cases. Radiology 2020; 296: E32-E40.

12. Liu H, Liu F, Li J, et al. Clinical and CT imaging features of the COVID-19 pneumonia: focus on pregnant women and children. J Infect 2020; 80: e7-e13.
13. Mahdavi A, Khalili N, Davarpanah AH, et al. Radiologic management of COVID-19: preliminary experience of the Iranian Society of Radiology COVID-19 Consultant Group (ISRCC). Iran J Radiol 2020; 17: e102324.

14. Yang W, Yan F. Patients with RT-PCR confirmed COVID-19 and normal chest CT. Radiology 2020; 295: E3.

15. Kim H, Hong H, Yoon SH. Diagnostic performance of CT and reverse transcriptase-polymerase chain reaction for coronavirus disease 2019: a meta-analysis. Radiology 2020; 201343.

16. Li Y, Xia L. Coronavirus disease 2019 (COVID-19): role of chest CT in diagnosis and management. AJR Am J Roentgenol 2020; 214: 12801286.

17. Chung M, Bernheim A, Mei X, et al. CT imaging features of 2019 novel coronavirus (2019-nCoV). Radiology 2020; 295: 202-207.

18. Guan WJ, Ni ZY, Hu Y, et al. Clinical characteristics of coronavirus disease 2019 in China. N Engl J Med 2020; 382: 1708-1720.

19. Ng MY, Lee EY, Yang J, et al. Imaging profile of the COVID-19 infection: radiologic findings and literature review. Radiology: Cardiothorac Imag 2020; 2: e200034.

20. Hope MD, Raptis CA, Henry TS. Chest computed tomography for detection of coronavirus disease 2019 (COVID-19): don't rush the science. Ann Intern Med 2020; 173: 147-148.

21. Hope MD, Raptis CA, Shah A, et al. A role for CT in COVID-19? What data really tell us so far. Lancet 2020; 395: 1189-1190.

22. ACR recommendations for the use of chest radiography and computed tomography (CT) for suspected COVID-19 infection. Radiology ACo 2020. Available from: https://www.acr.org/Advocacy-and-Economics/ ACR-Position-Statements/Recommendations-for-Chest-Radiography-and-CT-for-Suspected-COVID19-Infection. 